\title{
Elasto-viscoplasticity Behaviour of a Structural Adhesive Under Compression Loadings at Low, Moderate and High Strain Rates
}

\author{
D. Morin · G. Haugou $\cdot$ F. Lauro $\cdot$ B. Bennani $\cdot$ \\ B. Bourel
}

Received: 19 December 2014/ Accepted: 10 February 2015/Published online: 18 February 2015

(C) Society for Experimental Mechanics, Inc 2015

\begin{abstract}
Crashworthiness performance of automotives by introducing new materials is of high interest based on the global mass' reduction of cars. Bonded techniques are tested at present with respect to the reduction of the mass/ energy's ratio combined with recycling processes. In this study, a compression test program is performed on a bulk structural adhesive under a very large range of strain rates, thus $[0.1 ; 5000] / \mathrm{s}$. Uniaxial compression tests at 0.1 and 50/s are achieved using a high-speed hydraulic testing machine. A split Hopkinson bar' device made of PA66 is experimented so as to characterize soft materials in the upper domain of strain rates, thus $[500 ; 5000] / \mathrm{s}$. A special mold is used for the preparation of the adhesive plates. Two sheets are prepared (thickness $4 \mathrm{~mm}$ with $1 \mathrm{MPa}$ pressure and $6 \mathrm{~mm}$ with $4 \mathrm{MPa}$ pressure) in order to consider the effect of the pressure during the curing process. Water jet technique is used to extract cylindrical samples from the plates with accurate dimensions. The identification of the compression behaviour of the structural adhesive is lead with objective to find material parameters of a modified G'sell model for finite element modeling of crashworthiness.
\end{abstract}

Keywords Split Hopkinson bar - Toughened adhesive . Stress-strain curves $\cdot$ Behaviour modelling

D. Morin

Structural Impact Laboratory (SIMLab), Faculty of Engineering Science and Technology, Centre for Research-based Innovation, NTNU, 7491 Trondheim, Norway

G. Haugou · F. Lauro $(\bowtie) \cdot$ B. Bennani - B. Bourel

LAMIH, CNRS UMR 8201, University of Valenciennes and

Hainaut Cambrésis, 59313 Valenciennes, France

e-mail: franck.lauro@univ-valenciennes.fr

\section{Introduction}

The use of high strength adhesives in structural parts of automotives is of high interest for designers and engineers. This assembly technique gives the possibility to join any sort of material in almost configuration with particular interest regarding mass and cost reduction opportunities proposed on new generation of cars. Recently, a new generation of adhesives has been developed by the chemical industry in order to improve the performance of adhesives under dynamic loadings. These adhesives are based on an epoxy matrix modified by addition of polymer nodules affecting plasticity and other mechanical properties such as visco-elasticity, visco-plasticity, Bauschinger effect and damage evolution during plasticity $[1,2]$. This modification of the matrix also affects the failure behaviour by delaying the crack propagation into the matrix and then improving the fracture toughness [3, 4]. The mechanical behaviour of the adhesive is then close to polymer behaviour with a viscoelastic and/or a viscoplastic part. This behaviour is also hydrostatic pressure dependent and thermal dependent. This leads to complex behaviours which could be described by different models as non-linear viscoelasticity [5] or viscoplasticity [6] alone or coupled elasto-viscoplasticity [7-9]/viscoelasticity-viscoplasticity [10]. In the present study, the identification of the BETAMATE $1496 V^{T M}$ adhesive behaviour, produced by Dow Automotive, is done under compression loading based on an elasto-viscoplastic model.

In order to describe the mechanical responses of adhesives, different tests devices are suggested, and the scientific literature highlights two strategies:

- Experimental tests programs based on elementary assemblies (butt joint for tensile/compressive loadings, 
single or double lap joint for shear loading and scarf joints for mixed loadings [11, 12]). These tests are mostly proposed by the scientific review $[13,14]$, but a non-uniform stress distribution inside the bonded joints is generally involved.

- Experimental tests programs on bulk specimens available on condition that the behaviour of the adhesive has no dependency regarding the thickness of the samples [15].

Considering crashworthiness situations, the main point is the failure of the adhesive which changes the kinematic of the collapse. To predict the failure, the stress states must be well known when considering cohesive failure which is the case for this adhesive and then could be used in cohesive element formulation [16, 17]. The objective of this paper is then focusing on the identification on the behaviour of the adhesive under compression loading on various strain rates. The second strategy is then more interesting for an accurate description of the behaviour law of the adhesives. The adhesive is a polymer and then temperature dependent. For bonded joints, the influence of the initial temperature is highlighted by various authors and a modification of the behaviour of the adhesive in terms of strength and failure strain is observed. Ductile behaviour is obtained at high temperature with low strength and on contrary brittle behaviour at low temperature with high strength [18-20]. In this approach, only the room temperature is considered and the temperature increase due to mechanical deformation is taken intrinsically into account through the experimental data obtained by digital image correlation [21]. The increase of the temperature by the mechanical deformation is supposed to be low and will not modify strongly the behaviour of the adhesive. Mechanical properties of adhesive are influenced a lot by temperature, time of curing and pressure level applied during curing phases leading particularly to creation of voids in the epoxy matrix [22, 23]. The authors have performed an extended tests program based on bulk specimens extracted from plates manufactured at two pressure levels. One objective is to establish if the process has modified significantly the material responses.

A large range of strain rates is investigated from 0.1 up to 5000/s using high speed machine (low and medium strain rates), associated with electro-optical extensometer and 3D Digital Image Correlation technique, and split Hopkinson pressure bar technique (high strain rates) coupled with electro-extensometer only. The present paper provides results on experimental stress-strain responses under compression and material parameters of the corresponding modified G'Sell's model [24] taking into account strain rate sensitivity for the BETAMATE $1496 \mathrm{~V}^{T M}$ adhesive. The behaviour model chosen in this study is a phenomenological model which could be used easily in the context of a finite element code with a non associative plasticity and damage model to take into account the complex behaviour of the adhesive [7]. Volumetric strain variation is also obtained and is as the same order as in tension for polymer [16, 25].

\section{Experimental Procedure}

\section{Description of the Experimental Set Up}

The material responses of the considered adhesive are obtained using two experimental devices to cover a large range of strain rates, thus $0.1-50 / \mathrm{s}$ using a high speed testing machine and 500-5000/s by means of the Split Hopkinson bar test.

\section{High Speed Testing Machine}

A high speed testing machine (Instron VHS65/20-capacities: $65 \mathrm{kN}$, speed range: $1 \mathrm{~mm} / \mathrm{s}-20 \mathrm{~m} / \mathrm{s}$ ) is set to perform compression tests using special platens designed for reduced size of samples (Fig. 1a). A set of two piezoelectrical load cells (Fig. 1a-Kistler 9343) are mounted on the compression set-up with a calibrated measurement range close to $7 \mathrm{kN}$ (resolution $35 \mathrm{~N}$ ) and an electro-optical extensometer (Fig. 1b-Rudolph XR200-bandwidth $250 \mathrm{kHz}$ ) is prepared so as to ensure the measurement of the current shortening of the specimen up to large strains (measurement range $10 \mathrm{~mm}$-resolution $10 \mu \mathrm{m}$ ). Here, the description of the high-speed machine is omitted due the well knowledge of this device where natural frequencies disturbances have been widely underlined in the literature. In the present case, the natural frequency of the device is equal to $9 \mathrm{kHz}$ using platens where reduced mass and high stiffness are combined. For the present compression tests, a maximum velocity close to $0.3 \mathrm{~m} / \mathrm{s}$ is finally applied to limit inertia effects on the samples and natural disturbances of the set platens/load cell at the upper part of the device. Figure 2 depicts the forces' equilibrium obtained for a compression test performed at $0.3 \mathrm{~m} / \mathrm{s}$. It reveals, that no ringing oscillations occurred in the system and that the inertia effects are negligible. Compression tests over $1 \mathrm{~m} / \mathrm{s}$ are permitted on condition that stiff plates are positioned at $90^{\circ}$ and mounted on columns are depicted in Fig. 1b. The jack is then guided in the direction of the compression loading. When the current force increases dangerously a fuse located in the system breaks so as to protect the measurement devices and the hydraulic system.

\section{Nylon Split Hopkinson Bar}

For the upper range of strain rates, a set of PA66 cylindrical bar are used assuming elastic pulses separation along 
Fig. 1 a Location of the sample between platens (in blue), b high speed machine and measurement devices (load and displacement) (Color figure online)
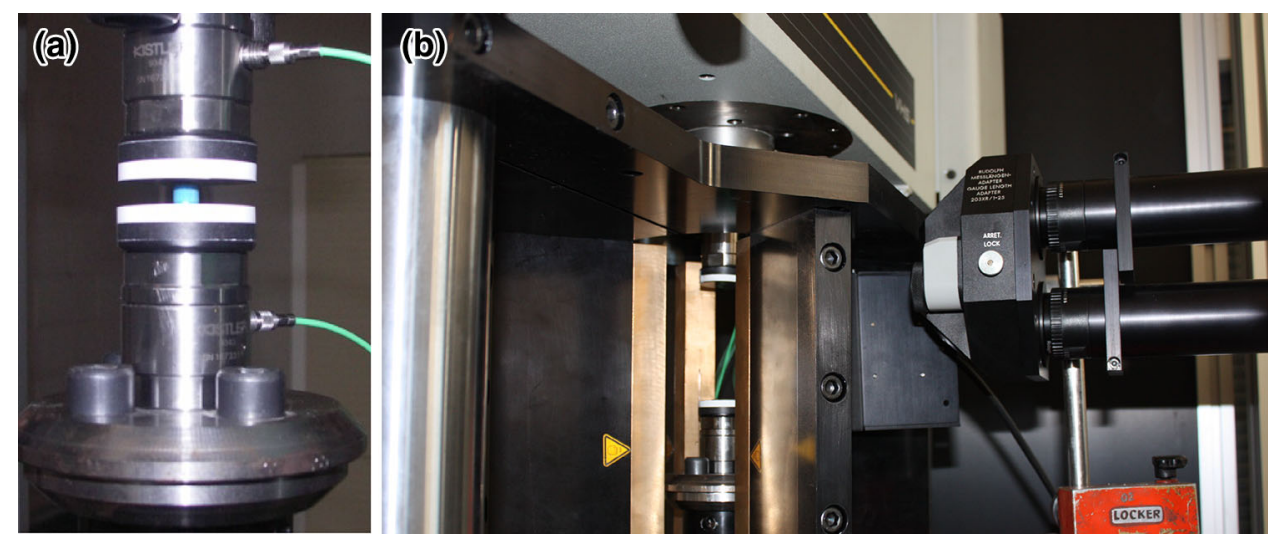

Fig. 2 Forces' equilibrium for a compression test performed at $0.3 \mathrm{~m} / \mathrm{s}$

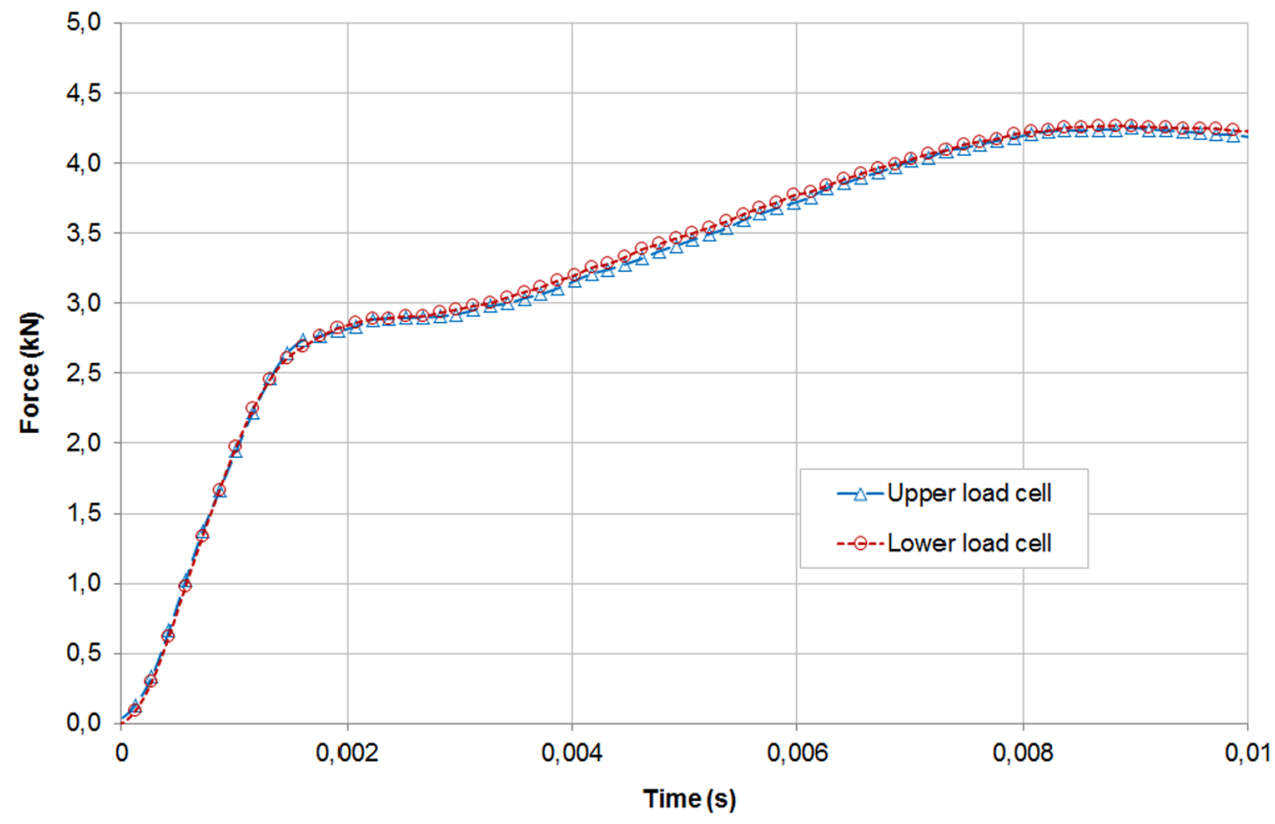

finite cylindrical bar. The configuration of the device is given in a schematic representation (Fig. 3a). The calculation of the material responses is determined on the basis of raw signals collected from full strain bridges bonded along input and output bar so that no superposition of the elastic incident and reflected pulses can occur during the test. For that, a Lagrangian diagram is needed in order to confirm the dimension of the bar as a function of the length of the projectile and the wave's speed of the elastic pulses. Here, the Split Hopkinson pressure bar device is composed of a set of bas with a diameter calibrated to $40 \mathrm{~mm}$ and a total length of each bar equal to $3 \mathrm{~m}$ (Fig. 3b). On the basis of the overall length of the device, the full-strain bridge cemented on the input bar is located at mid-length of the bar, and the full-strain bridge of the output bar is located at $400 \mathrm{~mm}$ at the interface with the sample. The measurement bar are aligned along a rigid frame made of aluminum so as to confer good parallelism conditions at the location of the sample (Fig. 3c). The loading of the sample is initiated when the incident pulse $\varepsilon_{I N C}(t)$ - generated during the impact of the projectile with the incident bar-is separated into two complementary pulses called $\varepsilon_{R E F}(t)$ and $\varepsilon_{T R A}(t)$ (reflected and transmitted pulses) propagating in the input and output bar, respectively. The history of the mentioned elastic pulses is finally converted to strain-stress relations (engineering and true data assuming Poisson' ratio correction) using classical governing equations detailed hereafter.

For many applications on Hopkinson bar devices, the constitutive material and the diameter of the bar are chosen so as to access to raw signals with acceptable amplitudes depending on the properties of the tested sample (natural 
(a)
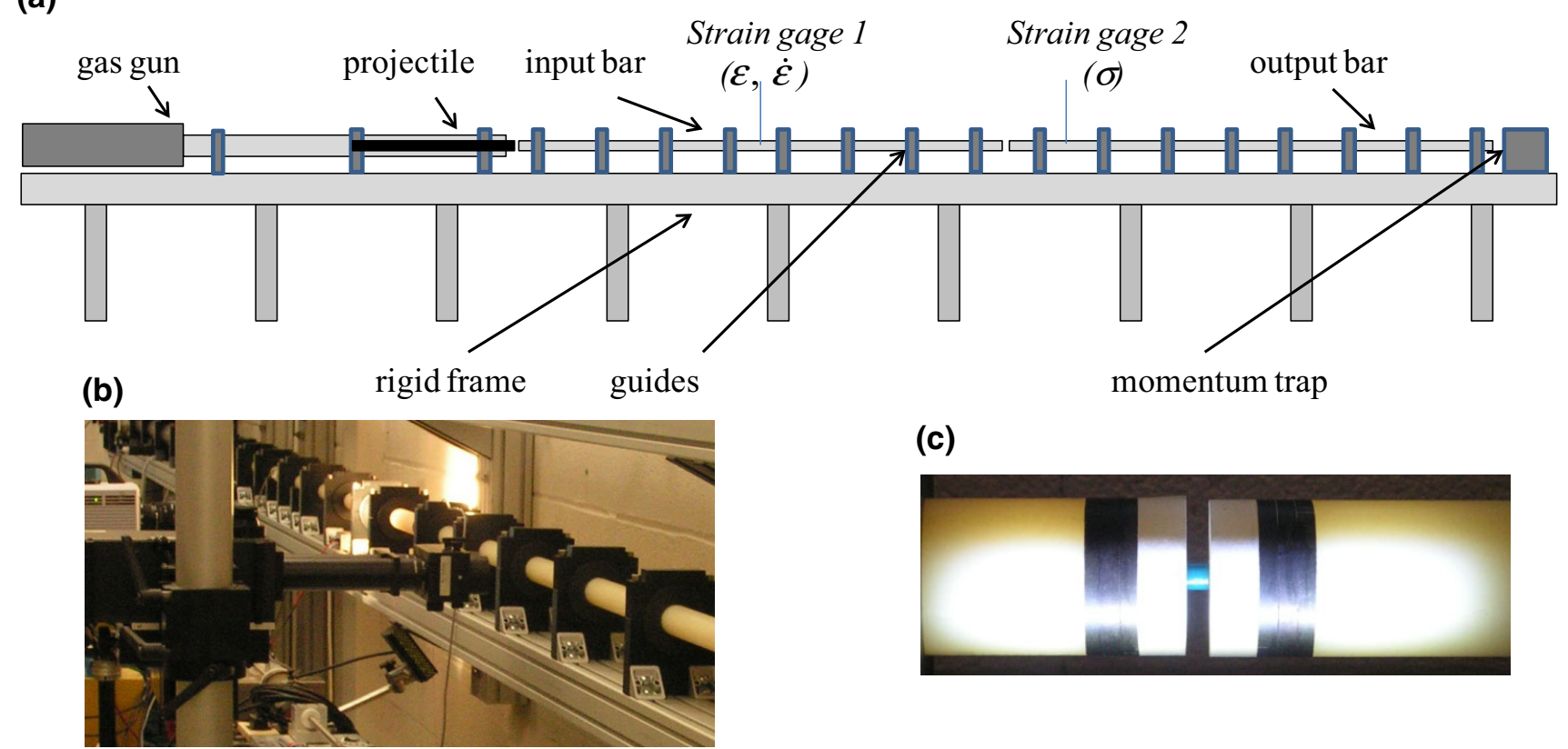

(c)

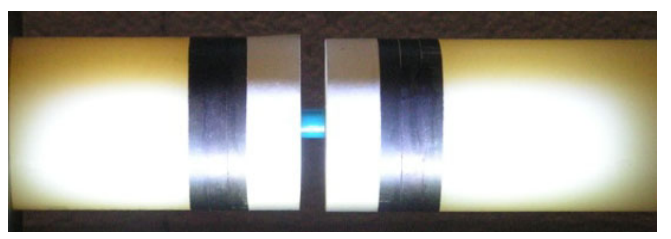

Fig. 3 a Scheme of the nylon compression bar device (overall length $10 \mathrm{~m}$ ). b PA66 bar for dynamic compression tests. c Location of the sample (in blue) (Color figure online)

amplifications of the transmitted signal are preferred compared to electrical amplifications applied on ampliconditioning systems). As polymer materials are tested, the natural amplitude of the output signal is reduced due to low level of stress state. In these conditions, measurement bar with low elastic properties are chosen so that a natural amplification of the signals is obtained. The length of the projectile (called L) determines also the duration time $\tau$ of the incident pulse with respect to the elastic wave velocity $C$ (Eq. 1).

$\tau=\frac{2 \cdot L}{C}$

For materials having high ultimate strain, a long duration time for the incident pulse is required (Eq. 2). Here, the projectile is made of Polyamid so that a length equal to $1 \mathrm{~m}$ ensures a duration time of the incident pulse close to $1.26 \mathrm{~ms}$. In these conditions, the material response of the sample with a total strain $\varepsilon(t)$ over 0.6 can be obtained at a mean strain rate $\dot{\varepsilon}(t)$ around $500 / \mathrm{s}$.

$\varepsilon(t)=\dot{\varepsilon}(t) \cdot \tau$

As a visco-elastic material is used here for the cylindrical bar, complementary calculations are lead to consider correction of dispersions along the bar as well as viscoelasticity of the constitutive material of the measurement bar [26]. The governing equations-required to access to the nominal stress-strain responses-are here reminded (Eqs. (3)-(6) expressed according to the elastic wave's formulation [27]). DAVID ${ }^{\odot}$ software developed by Gary and Degreef [28] is used to compute stress-strain relations on the basis of the three elementary elastic pulses $\varepsilon_{I N C}(t), \varepsilon_{R E F}(t), \varepsilon_{T R A}(t)$ detected during each test:

$\dot{\varepsilon}_{E n g}(t)=\frac{v_{I N}(t)-v_{\text {OUT }}(t)}{l_{S}}$

where $v_{I N}(t)$ and $v_{\text {OUT }}(t)$ are respectively the input and output bar' speed and $l_{S}$ is the initial gage length of the sample. $\dot{\varepsilon}_{E n g}(t)$ is the engineering strain rate. $\varepsilon_{E n g}(t)$ is the engineering strain finally obtained using a single integration as a function of time.

$v_{I N}(t)=C_{I N} \cdot\left(\varepsilon_{I N C}(t)-\varepsilon_{R E F}(t)\right)$

where $\varepsilon_{I N C}(t)$ and $\varepsilon_{R E F}(t)$ are respectively the amplitude of the incident and reflected pulses propagating in the input bar. $C_{I N}$ is the wave speed of the incident and reflected pulses propagating in the input bar.

$v_{\text {OUT }}(t)=C_{\text {OUT }} \cdot \varepsilon_{T R A}(t)$

where $\varepsilon_{T R A}(t)$ is the amplitude of the transmitted pulse and $C_{\text {OUT }}$ is the wave speed of the transmitted pulse propagating in the output bar.

$\sigma_{E n g}(t)=\frac{S_{\text {OUT }} \cdot E_{\text {OUT }}}{S_{S}} \cdot \varepsilon_{T R A}(t)$

where $S_{O U T}$ and $S_{S}$ are respectively the gage section of the output bar and the sample. $E_{\text {OUT }}$ is the elastic modulus of the output bar. $\sigma_{E n g}(t)$ is the current stress calculated in the sample. 
The current displacements at input/output bar and the shortening of the specimen are obtained with a first order integration in function of time applied on Eqs. (4) and (5), respectively. This statement is also admitted for the calculation of the current strain on the basis of Eq. (3).

As compression tests are performed using PA66 bar for samples with small diameters and similar flow stress, a punching effect can be observed [29]. In practice, a local displacement is induced during the elastic phase of the dynamic loading. This occurs at the interfaces of the bar with the sample and has to be considered in the determination of the apparent elastic modulus of the sample at the early stage of the material response [28].

Finally, a set of two high-speed camera (Photron APX RS 3000) oriented with an angle close to $30^{\circ}$ with a frame rate of $50 \mathrm{f} / \mathrm{s}(1024 \times 1024$ pixels $)$ is used to realize 3D Digital Image Correlation calculations with objectives to extract full strains and strain rates fields. The measurements using non-contact technique are restricted to tests performed at $0.1 / \mathrm{s}$ (Fig. 4) at a first stage.

\section{Preparation of the Samples}

The preparation of bulk specimens of the BETAMATE $1496 V^{T M}$ adhesive has leaded the authors to define a special forming process so that plates of pure adhesive can be obtained. An aluminum mould with a rectangular form is designed to produce the plates. A Teflon film was previously spread to avoid adhesions during the curing cycle between the mould and the fresh adhesive stored in a cartridge. Mechanical properties of adhesives can be significantly affected by temperature and time of curing but also by the pressure level applied for the duration of the curing process $[22,23]$. For that, the authors have decided to control these parameters applied on the different plates with a heating press. Two pressure levels are applied on the plates during the curing phase, thus 1 and $4 \mathrm{MPa}$ respectively. Despite the special care given to the preparation of the plates, it remains difficult to obtain pore-free samples. The authors have then controlled the porosity with lightening through the plates. They have also quantified the size of pores which cannot be detected easily using the X-ray $\mu \mathrm{CT}$ technique. The greatest pore size is quantified close to $80 \mu \mathrm{m}$ so that these values will be considered as initial void for further FE simulations. After these controls, specimens are machined using a water jet cutting in the adhesive plates. This technique gives the opportunity to reduce the heating of small size samples as well as crack initiation encountered with classical machining techniques. Before the tests, the density of the constitutive material of the adhesive is determined using Archimede's principle and evaluated here close to $1175 \mathrm{~kg} / \mathrm{m}^{3}$. The authors have performed no heat-treatment to relieve residual stresses at the surface of the samples.

The proposed geometry respects the standard diameter to height ratio (Fig. 5a, b).

\section{Identification of Material Parameters}

Since global force-displacements measurements have revealed a reasonable repeatability, a set of 3 replicates per speed are performed. A set of 6 samples is then tested using the high speed machine with the mentioned dimensions. The range of velocity is equal to 1 and $300 \mathrm{~mm} / \mathrm{s}$ providing mean true strain rates close to 0.15 and $50 / \mathrm{s}$ respectively. The material responses are completed by dynamic compression testing of a set of 9 samples on the Split Hopkinson pressure bar device (SHPB) described previously for 3 different velocities. The typical response used for the determination of the strain-stress relations is illustrated in Fig. 6.

Figure 6 illustrates non perfect contact conditions between the stricker and the input bar. Indeed, a tail is added at the end of the incident pulse and the authors have checked that it has no effects on the amplitude of the reflected pulse. As a consequence, the tail is put to zero so as
Fig. 4 Configuration of the set of high-speed cameras $(1024 \times 1024$ pixels at $50 \mathrm{f} / \mathrm{s})$

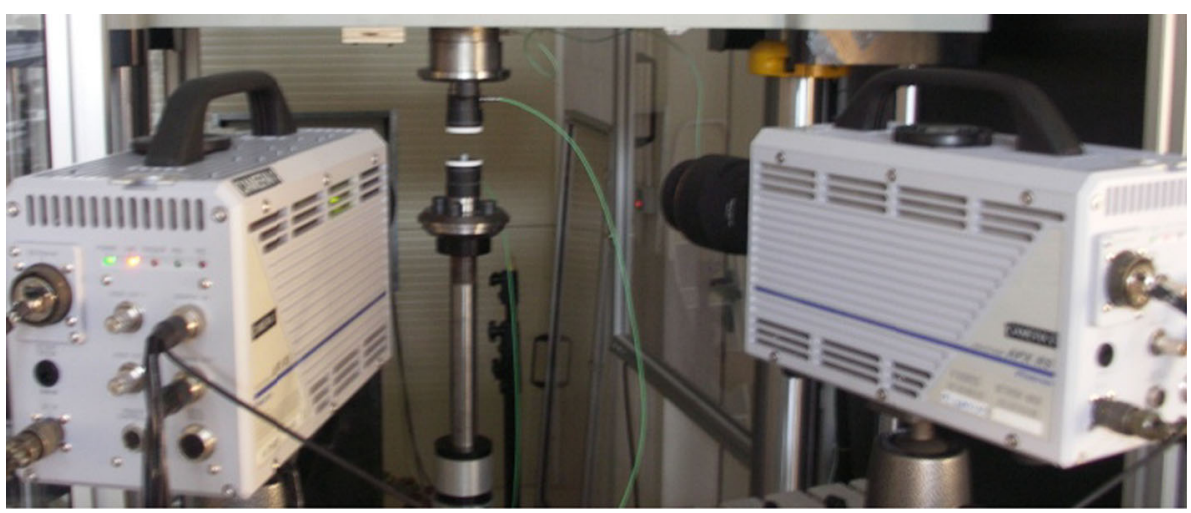


(a)

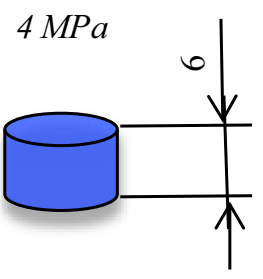

$\varnothing 7$ (b)

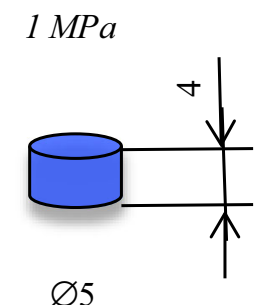

$\varnothing 5$

(in $\mathrm{mm}$ )

Fig. 5 a Samples from plate with $4 \mathrm{MPa}$ pressure, b samples from plate with $1 \mathrm{MPa}$ pressure

to consider a perfect separation in time of both pulses detected in the input bar.

When dealing with Hopkinson bar technique, the forces' equilibrium between input and output bar has to be checked to ensure the validity of the measurements. The input and output forces are determined by Eqs. 7 and 8, with:

$F_{I N}(t)=S_{I N} \cdot E_{I N}\left(\varepsilon_{I N C}(t)+\varepsilon_{R E F}(t)\right)$

$F_{\text {OUT }}(t)=S_{\text {OUT }} \cdot E_{\text {OUT }} \cdot \varepsilon_{\text {TRA }}(t)$

where $E_{I N}, E_{\text {OUT }}$ are the elastic modulus of the input and output bar, respectively.

As observed in Fig. 7, the equilibrium of the forces is obtained for the duration of the test, and further calculations are then permitted on the basis of the governing equations given in Eqs. 1-4:

Figure 7 illustrates also the ultimate plastic strain where the true strain rate assumed to be quasi-constant, thus here below 0.4 in true strain. Over, the current is increasing and then the true strain rate is decreasing since the loading principle of the device is not closed loop. Finally, the strain

rate value depending partially on the geometry of the sample, two levels of strain rates are covered, thus here:

- 600 up to 2500/s for samples with $6 \mathrm{~mm}$ thick (4 MPa),

- 1400 up to $5000 / \mathrm{s}$ for samples with $4 \mathrm{~mm}$ thick (1 MPa).

In Fig. 8 are illustrated samples before and after compression tests; the authors have previously lubricated each face of the sample using a high pressure lubricant $\left(\mathrm{MoS}_{2}\right)$ so as to:

- reduce friction effects,

- ensure homogenous strains and prevent from buckling troubles.

Validation of the True Stress-Strain Relations

The behaviour law determination depends on the longitudinal strain and the validity of the strain calculated after the test has to be checked. The longitudinal strain is only valid on condition that no buckling occurs during the test. The current shape of the cylindrical samples is monitored using 3D Digital Image Correlation with tests performed on the high-speed machine. As shown in Fig. 9, the shape of the specimen has revealed a cylindrical shape up to high plastic strains without global buckling for tests performed at $0.1 / \mathrm{s}$. The global calculations of longitudinal strains using the 3D Digital Image Correlation technique are then admitted up to the high plastic strains for the considered structural adhesive.

Once the calculation of the longitudinal strain is carried out, the computation of the current stress is then validated for the tests at medium and high strain rates. For the computation of the current stress on the Hopkinson bar
Fig. 6 Typical recording for the compression tests on bulk adhesive specimens

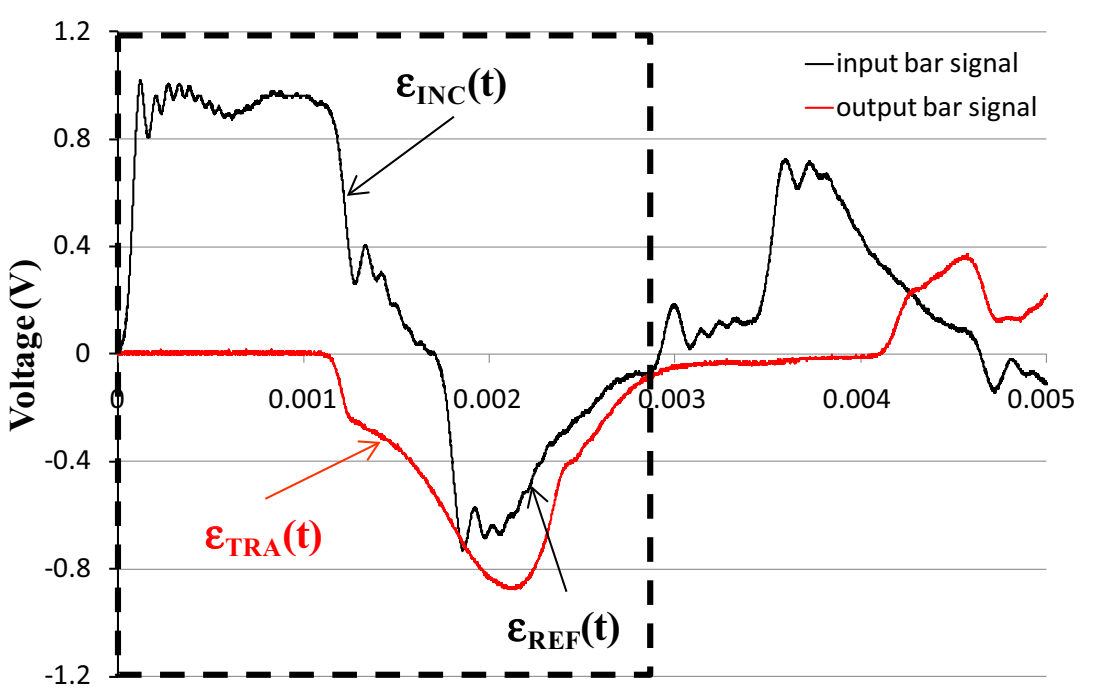

Time (s) 
Fig. 7 Forces' equilibrium at the interface of the bar and strain rate evolution in function of the total strain

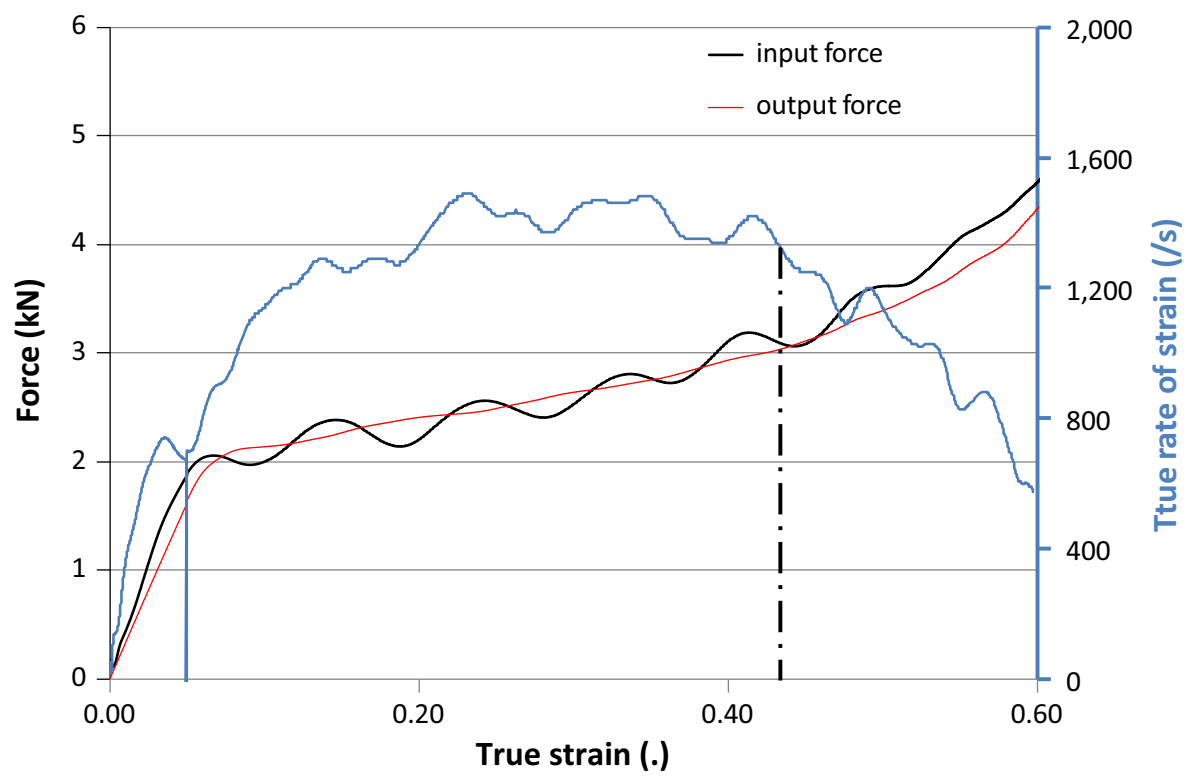

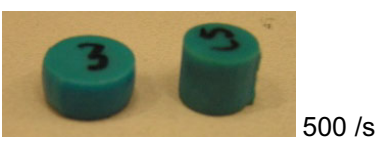

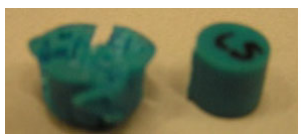

$1400 / \mathrm{s}$

Fig. 8 Geometry of the compression specimens before and after the test

tests, an additional step is needed. Longitudinal strains are not extracted directly from a global measurement but from the 1-D wave mechanics (Eq. (3), [27]). For these reasons, a comparison between total strains calculated from governing equations related to Hopkinson tests and measurements from an electro-optical extensometer (measurement range $10 \mathrm{~mm}$-resolution $10 \mu \mathrm{m}$ ) is given in Fig. 10. A good correlation between both techniques regarding the displacement/time responses at the input (IB) and output (OB) bar as well as the current shortening of the sample is then verified. The current strain-stress relations computed from the Hopkinson bar recordings is finally validated (Eqs. 3 and 6) and converted in true relations (Eqs. 9 and 11).

$$
\begin{aligned}
& \dot{\varepsilon}_{\text {True }}(t)=\frac{\dot{\varepsilon}_{\text {Eng }}(t)}{\dot{\varepsilon}_{\text {Eng }}(t)-1} \\
& \varepsilon_{\text {True }}(t)=\operatorname{Ln}\left(\varepsilon_{\text {Eng }}(t)-1\right) \\
& \sigma_{\text {True }}(t)=\sigma_{\text {Eng }}(t) \cdot e^{\left(-2 \cdot v \cdot \varepsilon_{\text {True }}(t)\right)}
\end{aligned}
$$

(a)

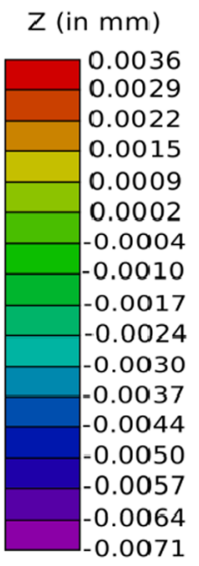

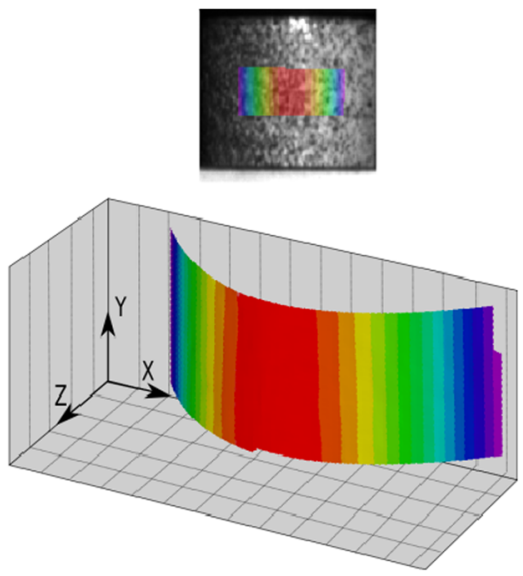

(b)

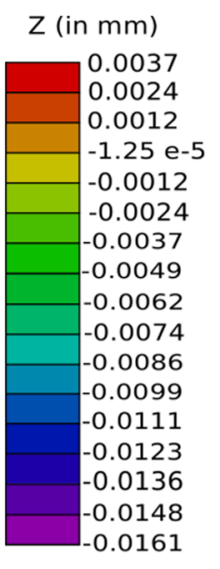

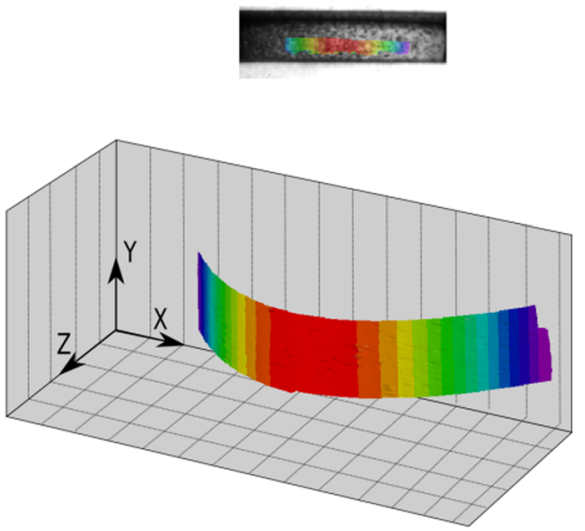

Fig. 9 Initial (a) and deformed (b) shapes of a cylindrical sample (at 0.10/s) 
Fig. 10 Comparison of the displacements' calculation (500/ s): electro-optical extensometer (thick line) and Hopkinson formulas (dotted line)

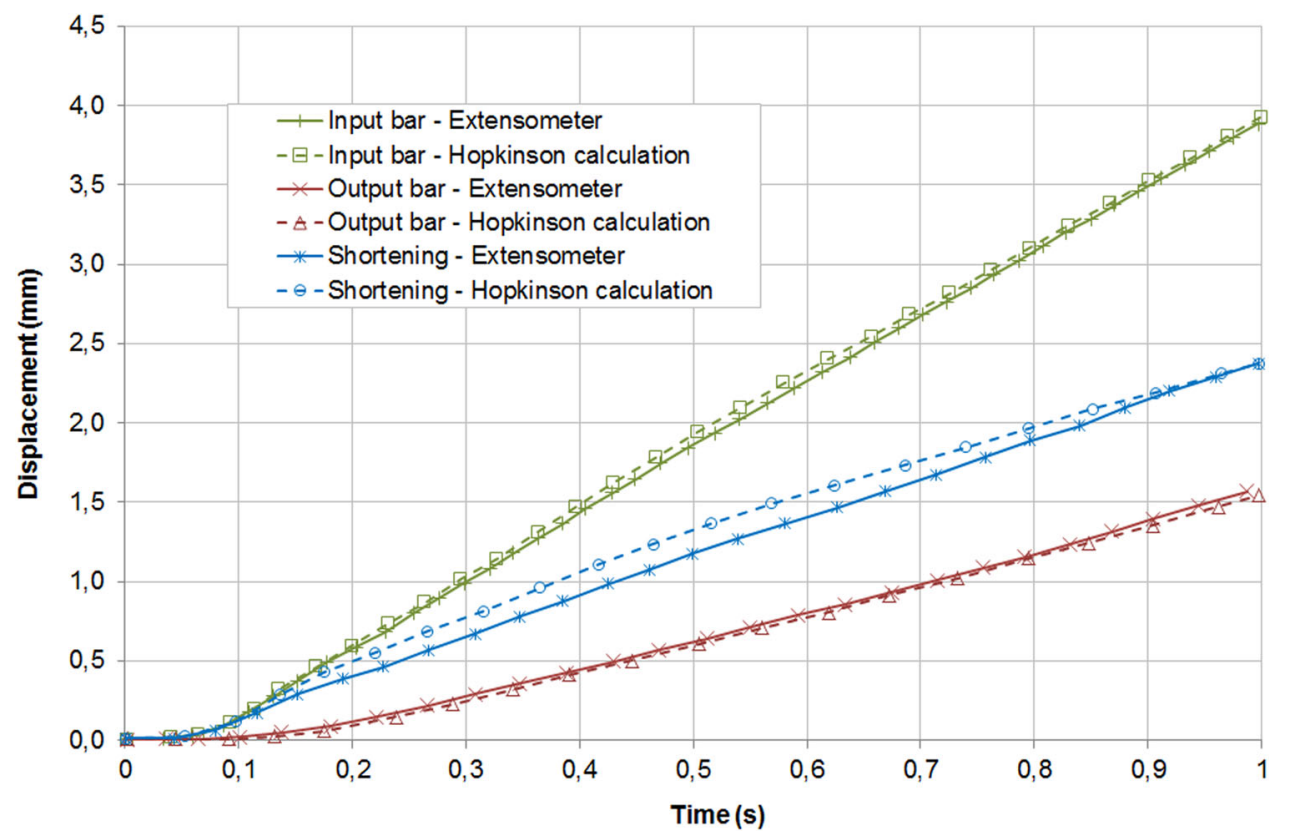

where $v$ is Poisson's ratio considered to be around $v=0.42$ [25].

\section{Experimental Behaviour Laws of Bulk Structural Adhesive}

The behaviour responses of the bulk adhesive under compression loading are high dependent on strain rate in elastic and plastic domains. Indeed, the apparent elastic modulus varies from 1.49 to $3.2 \mathrm{GPa}$ for specimens cured at the $4 \mathrm{MPa}$ curing pressure (Fig. 11a) and from 1.38 to $2 \mathrm{GPa}$ for those cured at $1 \mathrm{MPa}$ (Fig. 11b). Note that, the apparent elastic moduli values based on tests results performed on SHPB are determined considering the punching correction progress detailed by Safa [29]. As a satisfactory agreement is obtained on the calculation of shortening of the sample using governing equations and electro-optical extensometer technique (Fig. 9), the authors consider that the elastic modulus values are acceptable here. An upper limit of the apparent elastic modulus is observed for strain rate greater than 2000/s. The same observations can be made on the visco-plastic domain, True strain rate dependency and stress saturation are also found. Both stress-strain relations have underlined a structural hardening around 0.4 in true plastic strain (Fig. 11a, b).

The curing process has revealed significant differences in the stress level between the two series of samples at similar strains. This stress sensitivity is strain rate dependent since the gap between the 1 and $4 \mathrm{MPa}$ behaviour laws is of $10 \mathrm{MPa}$ for $50 / \mathrm{s}$ and $20 \mathrm{MPa}$ for $1500 / \mathrm{s}$, respectively (Fig. 12). Although curing pressure has already been addressed in the literature [27], the viscous effect of this sensitivity is not explained and might be due to the complex microstructure of the considered structural adhesive. The particles added in the matrix are one possible explanation of this sensitivity. The stress state created under higher pressure at the interface particle/matrix could lead to different local behaviours concerning the damage evolution.

A study is also carried out on the volume variation occurring during compression loadings. Indeed, this kind of phenomenon is well known for tensile loadings but suffers of a lack of knowledge for compression loadings. Some recent works [15] have shown that volume variation can also take place in compression for polymers. Using previous D.I.C. calculations, the volume variation is calculated by using the displacement in the $\mathrm{Z}$ direction (Fig. 9) of two points in the high of the cylinder. Considering that the cylinder stays a perfect cylinder as observed by the DIC analysis during the deformation, the volume variation is obtained by calculating the difference of the measured volume and the volume obtained under the incompressible hypothesis. The uniformity of the deformation observed by DIC could be of course discussed near contact surface as perfect sliding is not possible. In consequence, the volume variation calculated in this paper is probably over estimated. This statement must be checked by using for example $\mu \mathrm{CT}$ scan to observe the damage evolution as in [30]. A non-isochoric plasticity is then highlighted for toughened epoxy adhesive (Fig. 13). The plastic behaviour 
Fig. 11 a True stress-strain curves of samples with $4 \mathrm{MPa}$ curing pressure level-quasistatic and dynamic compression loadings, b true stress-strain curves of samples with $1 \mathrm{MPa}$ curing pressure level
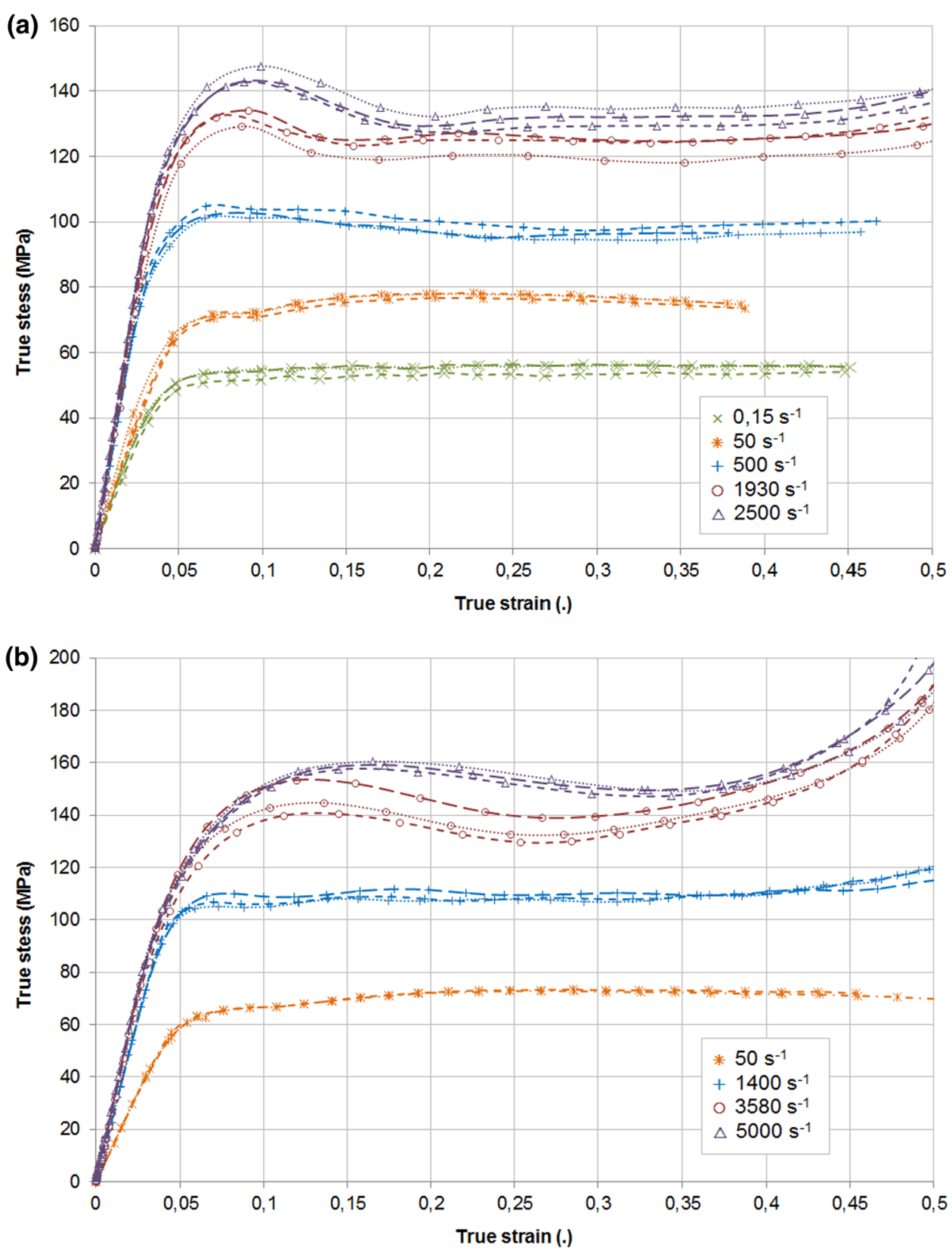

of toughened epoxy adhesive does not confirm any compaction (i.e. negative volumetric strains) but positive and then dilatants volumetric strains. This statement can also be linked to the complex microstructure of these adhesives and to the possibility of debonding between mineral and/or polymer charges and the epoxy matrix especially at the circumference of the cylinder where tension could be observed. Cracks generally start from the outer diameter perpendicularly to main circumferential deformation as shown Fig. 14 for two different deformation steps.

\section{Behaviour Laws Modeling}

Plastic behaviour of structural adhesives can be implemented in finite element codes with tabulated behaviour laws or using mathematical models. In terms of mathematical models, visco-plasticity is generally modelled in commercial finite element codes using Johnson-Cook or Cowper-Symmonds models. Nevertheless, these models are not able to reproduce the experimental behaviours observed for the adhesive. The evolution of the true stress as a 
Fig. 12 Comparison of true stress-strain relations for a set of samples tested at 50 and around 1700/s

Fig. 13 Evolution of volumetric strains during compression tests for $4 \mathrm{MPa}$ curing pressure level
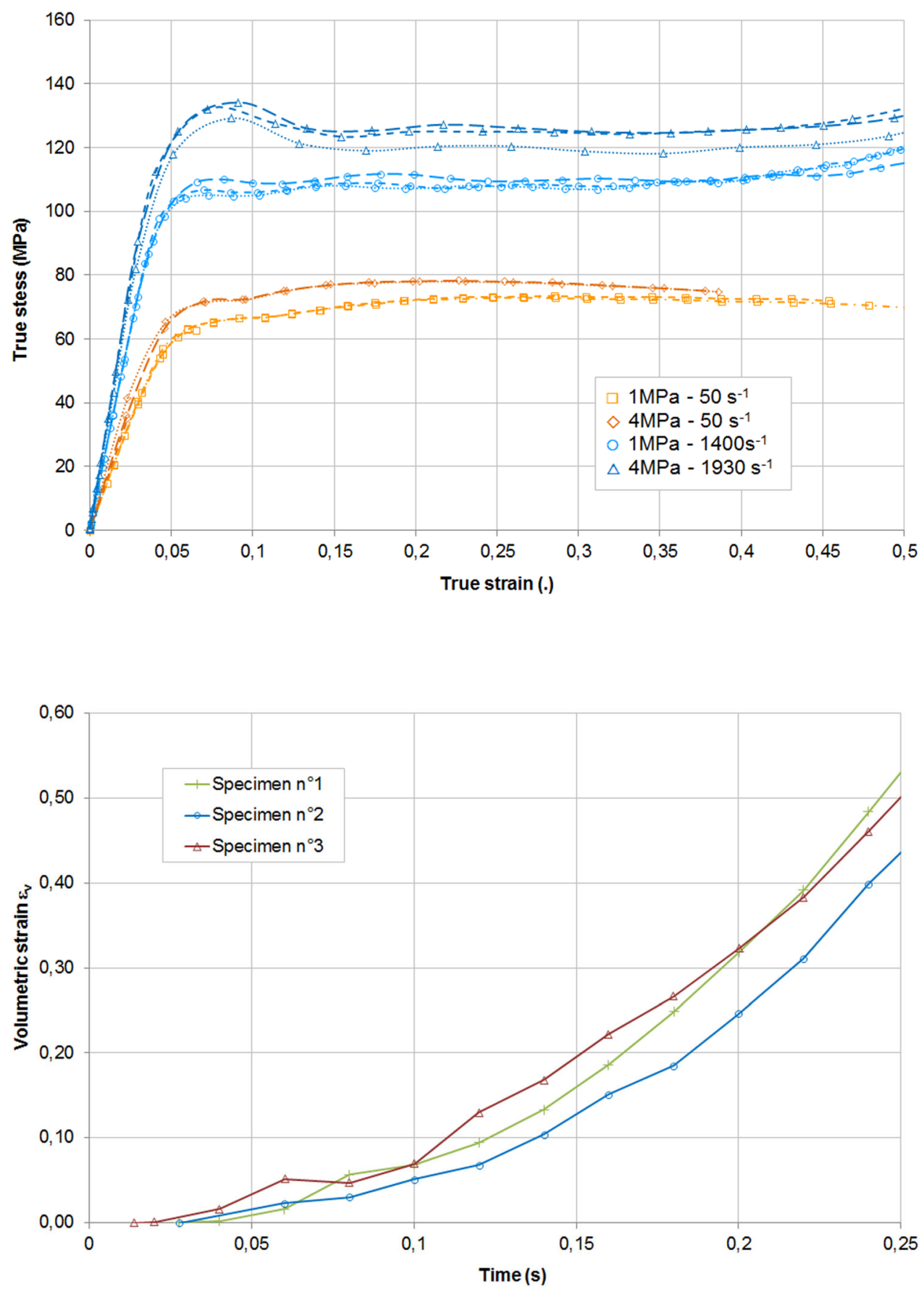

function of the true strain is not always the same at considered strain rates. To match as close as possible the experimental results, a strain rate dependency is introduced into the G'Sell description of the plasticity behaviour [24]. The modified G'Sell model is defined by the following expression:

$$
\begin{aligned}
\sigma= & \sigma_{y}(\dot{\varepsilon})+K \cdot\left(1-\exp \left(-w \cdot \varepsilon_{p}\right)\right) \\
& \cdot\left(1+h_{1}(\dot{\varepsilon}) \cdot \varepsilon_{p}+h_{2}(\dot{\varepsilon}) \cdot \varepsilon_{p}^{2}+h_{3}(\dot{\varepsilon}) \cdot \varepsilon_{p}^{3}\right)
\end{aligned}
$$

where $\sigma_{\mathrm{y}}$ defines the yield stress, $\varepsilon$ and $\varepsilon_{\mathrm{p}}$ are the true strain rate and the true plastic strain respectively, $K, w$ are ramping parameters and $h_{1}, h_{2}, h_{3}$ define the structural hardening. $\sigma_{\mathrm{y}}, h_{1}, h_{2}$ and $h_{3}$ are strain dependent.

The identification of these parameters is achieved by using a non-linear least squares technique coupled to a Levenberg-Marquardt algorithm. The common problem of this kind of parameters optimization is the uniqueness of 


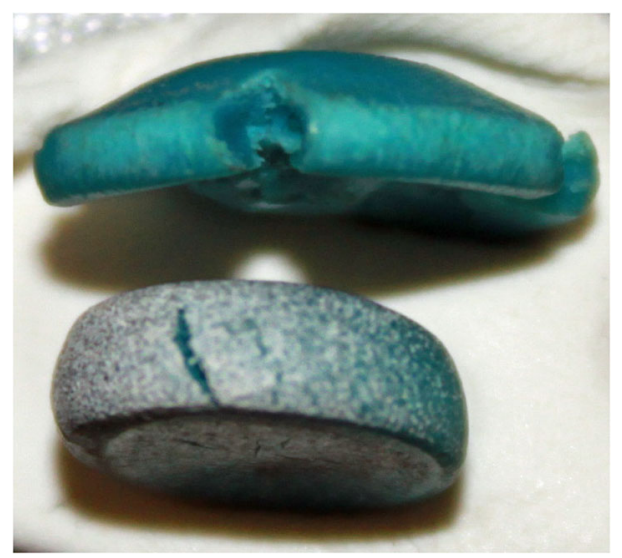

Fig. 14 Development of the crack propagation at the outer diameter of the cylinder (geometry Fig. 5a)

Table 1 Identified parameters for the 1 and $4 \mathrm{MPa}$ specimens

\begin{tabular}{lllll}
\hline True strain rate $(/ \mathrm{s})$ & $\sigma_{y}$ & $h_{1}$ & $h_{2}$ & $h_{3}$ \\
\hline $1 \mathrm{Mpa}$ & & & & \\
53 & 40 & 3.20 & -5.4 & 1.1 \\
1400 & 87 & 3.3 & -21 & 43.5 \\
3500 & 90 & 18.5 & -98 & 170 \\
5000 & 92 & 28.3 & -140 & 213 \\
$4 \mathrm{Mpa}$ & & & & \\
0.53 & 35 & 2.00 & -4.4 & 4.3 \\
53 & 51 & 3.40 & -4.4 & 15.4 \\
500 & 68 & 12.3 & -52 & 73 \\
1800 & 103 & 12.3 & -54.3 & 80 \\
2500 & 106 & 12.3 & -55.6 & 82 \\
\hline
\end{tabular}

the solution. To reduce this issue, the parameter identification is achieved through the following steps:

- Identification on the low strain rate behaviour law,

- Identification on the high strain rate behaviour law,

- Identification on the intermediate strain rates behaviour law.

For the intermediate strain rates, the parameters identified on the low and high strain rates behaviour laws are used as boundaries for the optimization algorithm. By using this kind of boundaries definition, monotonic evolutions of the parameters are assumed.

The results in terms of parameters identification are given in Table 1 for the specimen extracted from plates at 1 and 4 MPa pressure level. The ramping parameters $K$ and $w$ are equal to 25 and 69 respectively for the $1 \mathrm{MPa}$ specimens and 17.5 and 80 for the $4 \mathrm{MPa}$ ones. A power law dependent on the strain rate can be determined for the yield stress $\sigma_{y}$ and the $h_{1}, h_{2}$ and $h_{3}$ parameters.

An illustration of the resulted fitting of the proposed model is given in Fig. 15 for the $1 \mathrm{MPa}$ specimens. As expected, the effect of the curing pressure is not limited to the yield stress but to all the parameters.

\section{Conclusions}

The various applications of high-strength adhesives in transportation industries has lead the scientific community to identify mechanical properties of these materials in particular case of crashworthiness and impact of transportation frameworks. Structural adhesives give the
Fig. 15 Illustration of correlation between proposed visco-plastic model and experimental results for the $1 \mathrm{MPa}$ specimens

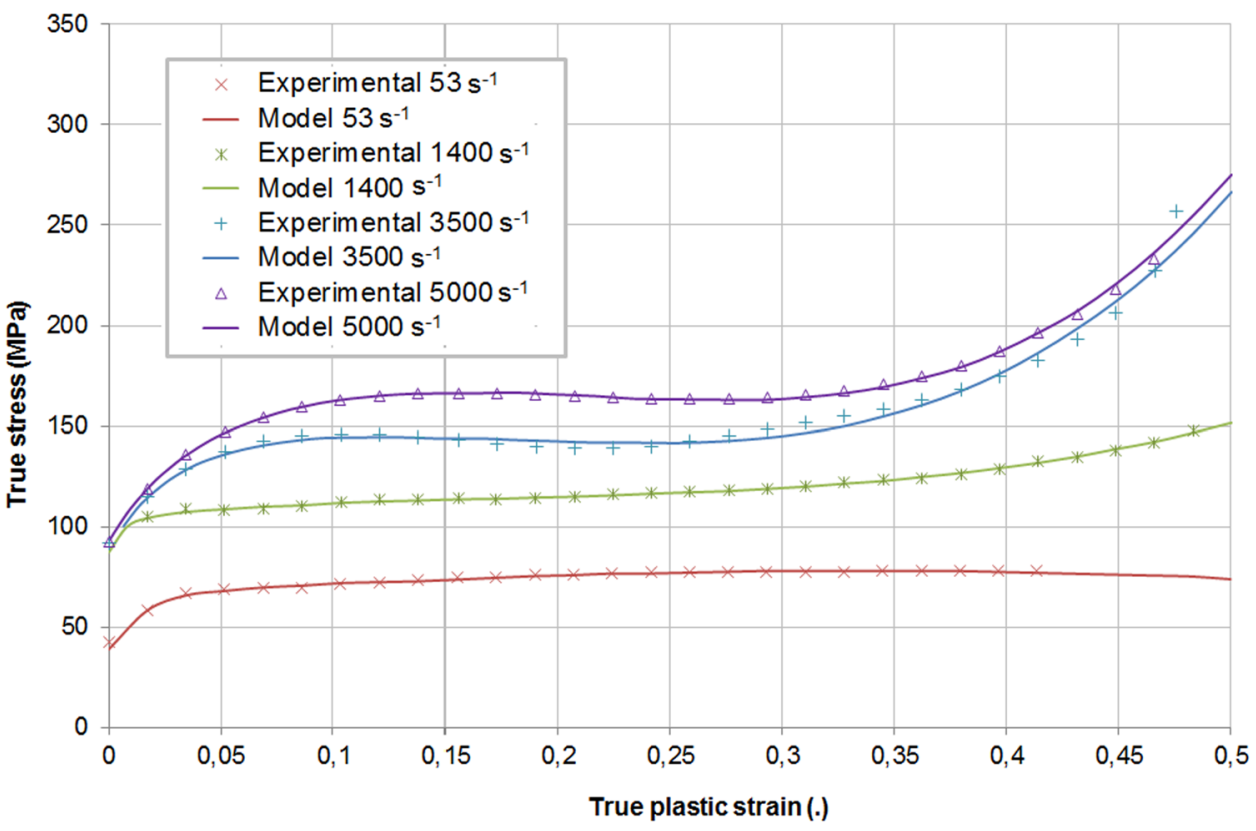


opportunity to join different classes of materials as a function of their mechanical properties and are in accordance with future environmental regulations (recycling, lightweight materials, etc.). Non-contact measurement techniques provide here stress-strain relations from quasistatic to dynamic loadings up to very large plastic strains. The present work provides a complete description of the evolution of the BETAMATE $1496 \mathrm{~V}^{\mathrm{TM}}$ adhesive for energy capabilities of automotive frameworks at moderate and high rates of loadings. The curing pressure has revealed to be an influencing parameter on the materials responses on bulk samples. To perform modelling of structural adhesive behaviour with finite element codes, an elasto viscoplastic model is proposed and parameters are identified.

Acknowledgments The present research work has been supported financially by the International Campus on Safety and Intermodality in Transportation (http://www.cisit.org), the Nord-Pas-de-Calais Region, the European Community, the Regional Delegation for Research and Technology, the Ministry of Higher Education and Research, and the National Centre for Scientific Research. The authors gratefully acknowledge also D. Lesueur for the preparation of the Hopkinson bar platform developed at the L.A.M.I.H. Laboratory.

\section{References}

1. Duncan B, Dean G (2003) Measurement and models for design with modern adhesives. Int J Adhes Adhes 23:141-149

2. Goglio L, Peroni L, Peroni M, Rossetto M (2008) High strain rate compressive and tension behaviour of an epoxy bi-component adhesive. Int J Adhes Adhes 28:329-339

3. Joudon V, Portemont G, Lauro F, Bennani B (2014) Experimental procedure to characterize the mode I dynamic fracture toughness of advanced epoxy resins. Eng. Fract. Mech 126: 166-177

4. Pearson RA, Yee AF (1993) Toughening mechanisms in thermoplastic-modified epoxies: 1. Modification using poly(phenylene oxide). Polymer 34:3658-3670

5. Badulescu C, Germain C, Cognard JY, Carrere N (2015) Characterization and modelling of the viscous behaviour of adhesives using the modified Arcan device. J Adhes Sci Technol 29:443-461

6. Pandey PC, Shankaragouda H, Kr Singh A (1999) Nonlinear analysis of adhesively bonded lap joints considering viscoplasticity in adhesives. Comput Struct 70:387-413

7. Balieu R, Lauro F, Bennani B, Delille R, Matsumoto T, Mottola E (2013) A fully coupled elastoviscoplastic damage model at finite strains for mineral filled semi-crystalline polymer. Int. J. Plast. 51:241-270

8. Jousset P, Rachik M (2014) Implementation, identification and validation of an elasto-plastic-damage model for the finite element simulation of structural bonded joints. Int $\mathbf{J}$ Adhes Adhes 50:107-118

9. Epee AF, Lauro F, Bennani B, Bourel B (2011) Constitutive model for a semi-crystalline polymer under dynamic loading. Int. J. Solids Struct. 48:1590-1599

10. Frank GJ, Brockman RA (2001) A viscoelastic-viscoplastic constitutive model for glassy polymers. Int J Solids Struct 38:5149-5164
11. Berry NG, d'Almeida JRM (2002) The influence of circular centered defects on the performance of carbone-epoxy single lap joints. Polym Test 21:373-379

12. You M, Yan Z, Zheng X, Yu H, Li Z (2007) A numerical and experimental study of gap length on adhesively bonded aluminium double-lap joint. Int J Adhes Adhes 27:696-702

13. de Morais AB, Pereira AB, Teixeira JP, Cavaleiro NC (2007) Strength of epoxy adhesive-bonded stainless steel joints. Int $\mathbf{J}$ Adhes Adhes 27:679-686

14. Derewonko A, Godzimirski J, Kosiuczenko K, Niezgoda T, Kiczko A (2008) Strength assessment adhesive-bonded joints. Comput Mater Sci 43(1):157-164

15. Delhaye V, Clausen AH, Moussy F, Hopperstad OS, Othman R (2010) Mechanical response and microstructure investigation of a mineral and rubber modified polypropylene. Polym Test. 29: 793-802

16. Morin D, Haugou G, Bennani B, Lauro F (2010) Identification of a new failure criterion for toughened epoxy adhesive. Eng. Fract. Mech. 77:3481-3500

17. Morin D, Bourel B, Bennani B, Lauro F, Lesueur D (2013) A new cohesive element for structural bonding modelling under dynamic loading. Int. J. Impact Eng. 53:94-105

18. Chiu WK, Chalkley PD, Jones R (1994) Effects of temperature on the shear stress-strain behavior of structural adhesive. Comput. Struct. 53:483-489

19. Badulescu C, Cognard JY, Créac'hcadec R, Vedrine P (2012) Experimental analysis of the temperature-dependent behaviour of a ductile adhesive under tensile/compression-shear loads. ICEM15, Porto/Portugal

20. Bed A, Malvade I, Biswas P, Schroeder J (2007) An experimental and analytical study of the mechanical behaviour of adhesively bonded joints for variable extension rates and temperatures. Int $\mathbf{J}$ Adhes Adhes 23:1-15

21. Lauro F, Bennani B, Morin D, Epee AF (2010) The SEE method for determination of behaviour laws for strain rate dependent material-application to polymer material. Int. J. Impact Eng. 37:715-722

22. Mengel R, Häberle J, Schlimmer M (2007) Mechanical properties of hub/shaft joints adhesively bonded and cured under hydrostatic pressure. Int J Adhes Adhes 27:568-573

23. da Silva L, Adams RD, Gibbs M (2004) Manufacture of adhesive joints and bulk specimens with high-temperature adhesives. Int $\mathbf{J}$ Adhes Adhes 24:69-83

24. G'sell C, Jonas J (1979) Determination of the plastic behaviour of solid polymers at constant true strain rate. Int $\mathrm{J}$ Mater Sci 14:583-591

25. Morin D, Haugou G, Bennani B, Lauro F (2011) Experimental characterization of a toughened epoxy adhesive under a large range of strain rates. J. Adhes. Sci. Technol. 25:1581-1602

26. Zhao H, Gary G, Klepaczko JR (1997) On the use of the viscoelastic Split Hopkinson Pressure bars. Int. J. Impact Eng. 19: 319-330

27. Gary G (2001) Some aspects of dynamic testing with waveguides. New experimental methods in materials dynamic and impact. Warsaw, pp. 179-222

28. Gary G, Degreef V (2008) DAVID ${ }^{\odot}$, Users manual version, Labview version. LMS Polytechnique, Palaiseau

29. Safa K, Gary G (2010) Displacement correction for punching at a dynamically loaded bar end. Int J. Impact Eng. 37:371-384

30. Balieu R, Lauro F, Bennani B, Haugou G, Chaari F, Matsumoto T, Mottola E (2015) Damage at high strain rates in semi-crystalline polymers. Int. J. Impact. Eng. 76:1-8 